\title{
O PRÉ-NATAL REALIZADO PELO ENFERMEIRO: A SATISFAÇÃO DAS GESTANTES
}

Thiago Luis de Andrade Barbosa ${ }^{1}$, Ludmila Mourão Xavier Gomes ${ }^{2}$, Orlene Veloso Dias ${ }^{3}$

RESUMO: O objetivo do estudo foi investigar a satisfação das gestantes sobre a Consulta de Enfermagem Pré-natal em uma unidade de Saúde da Família de Montes Claros/MG, Brasil. Trata-se de um estudo exploratório de natureza qualitativa realizado com oito gestantes cadastradas na área de abrangência da unidade em que realizaram a consulta de pré-natal com o enfermeiro da equipe. Os dados foram coletados por meio de entrevista e analisados com a técnica de análise do discurso. Os resultados mostraram satisfação com a Consulta de Enfermeiro no pré-natal; a humanização e a competência profissional do enfermeiro foram destacadas pelas gestantes, evidenciando qualidades como capacidade, atenção, paciência e dedicação. A partir dos discursos pode-se concluir que houve unanimidade em relação à percepção das mulheres acerca da satisfação do serviço e à aprovação do pré-natal realizado pelo enfermeiro.

PALAVRAS-CHAVE: Cuidado pré-natal; Enfermagem; Percepção; Satisfação do paciente.

\section{PRE-NATAL CONSULTATION BY NURSES: CLIENT SATISFACTION AMONG EXPECTANT MOTHERS}

\begin{abstract}
The aim of the study was to investigate the degree of satisfaction with nurse-led prenatal consultations among mothers-to-be in a family health centre in Montes Claros, Minas Gerais, Brazil. This qualitative study was exploratory in character, carried out with the assistance of eight pregnant women registered in the area covered by the health unit where they were to receive their prenatal consultation with the team nurse. Data was collected via interviews and analysed through discourse analysis. The results showed satisfaction with the prenatal nurse consultation; the humanization and professional competency of the nurse were highlighted by the clients, with qualities such as competency, attention, patience and dedication being cited. It can be concluded from the discourses that the women were unanimous in relation to their perception of the service and their approval of the prenatal consultation carried out by the nurse.
\end{abstract}

KEYWORDS: Prenatal care; Nursing; Perception; Patient satisfaction.

\section{PRENATAL REALIZADO POR EL ENFERMERO: LA SATISFACCIÓN DE LAS GESTANTES}

RESUMEN: El objetivo del estudio fue investigar la satisfacción de las gestantes sobre la Consulta de Enfermería Prenatal en una unidade de Salud de la Familia de Montes Claros/MG, Brasil. Es un estudio exploratorio de naturaleza cualitativa realizado con ocho gestantes cadastradas en área abarcada por la unidad en que realizaron la consulta de prenatal con el enfermero del equipo. Los datos fueron recogidos por medio de entrevista y analizados con la técnica de análisis del discurso. Los resultados revelaron satisfacción con la Consulta de Enfermero en el prenatal; la humanización y la competencia profesional del enfermero fueron destacadas por las gestantes, evidenciando cualidades, como capacidad, atención, paciencia y dedicación. Con base en los discursos, fue posible concluir que hubo unanimidad acerca de la percepción de las mujeres sobre la satisfacción del servicio y la aprobación del prenatal realizado por el enfermero.

PALABRAS CLAVE: Cuidado prenatal; Enfermería; Percepción; Satisfacción del paciente.

\footnotetext{
${ }^{1}$ Enfermeiro. Coordenador da Vigilância Epidemiológica e do Programa de Imunização da Secretaria Municipal de Saúde de Januária/MG.

${ }^{2}$ Enfermeira. Mestre em Ciências da Saúde. Doutoranda no Programa de Pós-Graduação em Enfermagem da Universidade Federal de Minas Gerais.

${ }^{3}$ Enfermeira. Mestre em Ciências da Saúde. Enfermeira da Prefeitura Municipal e Professora do Curso de Graduação em Enfermagem da Universidade Estadual de Montes Claros/MG.
}

\section{Autor correspondente:}

Thiago Luis de Andrade Barbosa

Secretaria Municipal de Saúde de Montes Claros

R. Barão de Cotegipe, 330 - 39400-388 - Montes Claros-MG-Brasil

Recebido: $29 / 04 / 10$

E-mail: tl_andrade@yahoo.com.br

Aprovado: 02/02/11 


\section{INTRODUÇÃO}

O interesse crescente pela satisfação dos usuários do setor saúde teve início na década de 70 , baseado em aspectos técnicos e estruturais da qualidade da atenção. Nas últimas décadas, essa temática tem sido considerada uma importante ferramenta na qualidade do cuidado, bem como provedora de informações confiáveis e adequadas sobre a opinião do público em relação aos serviços prestados, para o seu aprimoramento $^{(1)}$.

O setor saúde adota políticas para o resgate da qualidade, possível por meio da sua descentralização, possibilitando a renovação dos modelos e práticas de atenção e cuidado à saúde, garantindo assim, maior utilização e continuidade dos serviços, podendo inclusive interferir no aumento da satisfação do usuário. Neste contexto, situa-se a humanização da atenção à saúde, uma oportunidade de transformação cultural da gestão e das práticas desenvolvidas nas instituições de saúde ao adotar postura ética de respeito e acolhimento ao usuário, entendido como cidadão e não apenas consumidor do serviço de saúde ${ }^{(1)}$.

O Sistema Único de Saúde (SUS) é uma política pública avançada e tem como princípios, além do direito à saúde, o controle social, a integralidade e a equidade das ações. Apesar de existir desde 1988 promulgado pela Constituição Federal e regulamentado pela Lei Federal 8.080 de 1990 - seus princípios não foram imediatamente implantados, devido à estrutura política e econômica governamental dos serviços de saúde, a qual desfavorecia o atendimento das necessidades da população, uma vez que privilegiava $\mathrm{a}$ atenção à doença e a grupos restritos. A realização dos princípios do SUS requer, entre outros aspectos, a estruturação de um novo modelo assistencial, cujo foco de atenção esteja voltado para os determinantes das condições de saúde de uma população ${ }^{(2)}$.

Com a implantação da Estratégia Saúde da Família (ESF), o SUS passou a incorporar atividades de cunho mais coletivo e abrangente, com perspectivas de, juntamente com outros setores, gerar impacto positivo na qualidade de saúde e de vida da população. A ESF vem contemplar uma modalidade de atenção cuja finalidade principal é desenvolver ações de promoção da saúde do indivíduo, da família e da comunidade, bem como prevenir doenças e outros agravos, sem deixar de lado as ações de tratamento e reabilitação(3). Sob essa ótica, a ESF condiz com a proposta de humanização do setor saúde, uma vez que propõe mudanças no modelo assistencial tradicional a partir de ações voltadas para a atenção primária, centrada na família, por meio da equipe multiprofissional ${ }^{(1)}$.

A ESF propõe uma dinâmica para a estruturação dos serviços de saúde, promovendo uma relação dos profissionais mais próximos dos usuários, famílias e comunidades, asusmindo compromisso de prestar assistência integral e resolutiva a toda população. A comunidade tem acesso garantido por meio de uma equipe multiprofissional e interdisciplinar que presta assistência de acordo com as reais necessidades das pessoas, identificando os fatores de risco aos quais elas estão expostas e neles intervindo de forma apro$\operatorname{priada}^{(4)}$.

Os serviços de saúde vêm passando por mudanças, no que diz respeito a estruturação e organização, com objetivo de construir um modelo de atenção à saúde mais justo, equânime, democrático, participativo e que tenha como prerrogativas básicas os princípios da humanização da assistência, promoção e prevenção em saúde ${ }^{(5)}$.

Nesse sentido, os profissionais da área da saúde, entre eles o enfermeiro, devem estar sensibilizados para a humanização da assistência prestada à clientela, tal como proposto pelo Programa de Humanização do Pré-natal e Nascimento. O enfermeiro deve compreender os seus fundamentos e a importância de humanizar e qualificar a atenção à gestante, a fim de obter sua maior adesão ao pré-natal, garantindo qualidade na assistência e melhores resultados obstétricos e perinatais com mãe e recém-nascido saudáveis.

A Consulta de Enfermagem, na atenção primária à saúde, é realizada de acordo com o roteiro estabelecido pelo Ministério de Saúde e pela Secretaria de Estado da Saúde ${ }^{(6-7)}$ e é amparada pela Lei do Exercício Profissional ${ }^{(8)}$.

Estudos relacionados à assistência pré-natal evidenciam que a Consulta de Enfermagem tem sofrido, ao longo do tempo, transformações em sua concepção, metodologia e, principalmente, sua inserção nos serviços de saúde, transitando para o prestígio e aceitação do profissional no seu fazer e assistir. A Consulta de Enfermagem proporciona a orientação de medidas favoráveis que visam à abordagem apropriada das necessidades peculiares das mulheres com quem os profissionais interagem em consultas no pré-natal, nas unidades básicas de saúde. A comunicação, neste contexto, é um recurso indispensável para a assistência à saúde, com vistas ao estabelecimento de confiança e a vinculação do usuário ao profissional, e 
consequentemente, ao serviço de saúde ${ }^{(9)}$.

O trabalho da Enfermagem está centrado no cuidado e tem como sujeito o cliente. Dessa forma, o profissional tem o dever de participar do processo de avaliação do "fazer da Enfermagem" de forma a contribuir para a melhoria da assistência prestada nos serviços de saúde, inclusive na ESF. Diante disso, é necessário conhecer a percepção das gestantes quanto à Consulta de Enfermagem pré-natal realizada no âmbito da atenção primária, como forma de detectar os fatores relacionados à satisfação e/ou insatisfação desse grupo. Sendo assim, torna-se indispensável à Enfermagem estudos que abordem o reconhecimento do seu trabalho e competência no âmbito da ESF, especificamente no atendimento às gestantes com pré-natal de risco habitual, visto que a percepção do usuário é também importante para as profissões. Assim, o presente estudo teve por objetivo analisar a satisfação das gestantes sobre a Consulta de Enfermagem pré-natal realizada em uma Unidade de Saúde da Família no Município de Montes Claros/MG.

\section{METODOLOGIA}

Tendo em vista o objetivo desta pesquisa, optouse por realizar um estudo exploratório de natureza qualitativa. O local escolhido para a sua realização foi uma Unidade de Saúde da Família, localizada na Região Sul do Município e que está dividida em seis microáreas que englobam os bairros Maria Cândida, Conjunto José Carlos de Lima e o Conjunto Havaí. Abrange 740 famílias cadastradas, conforme dados do Sistema de Informação da Atenção Básica (SIAB) do mês de abril de 2008. Nessa unidade são realizados grupos educativos com gestantes, além das consultas de prevenção ao câncer de colo de útero e a Consulta de Enfermagem pré-natal.

No estudo, foi adotada a abordagem qualitativa, que permite a abrangência da realidade social para além do que pode ser observado e quantificado. As Ciências Sociais, as quais se preocupam com os significados, têm ganhado força na atualidade, a partir da introspecção do homem e da observação de si mesmo como ator social, ressaltando questões que, até recentemente, passaram despercebidas ${ }^{(10)}$.

O universo da pesquisa foi constituído por 8 mulheres que tiveram suas consultas de pré-natal realizadas por enfermeiro da equipe e que aceitaram participar espontânea e formalmente da pesquisa.

O levantamento do universo de pesquisa foi feito segundo os critérios de inclusão: 1) ter idade igual ou superior a 18 anos; 2) ser residente na área de cobertura da unidade Saúde da Família; 3) ter pré-natal classificado como de risco habitual e 4) ter o pré-natal acompanhado pelo enfermeiro da Unidade de Saúde da Família.

A coleta de dados ocorreu entre abril e maio de 2008 , por meio de entrevista semiestruturada, aplicada individualmente, a partir de uma pergunta norteadora: "Qual a sua opinião/satisfação sobre a consulta de enfermagem pré-natal?". O roteiro semiestruturado permite combinar perguntas abertas e fechadas, na qual o entrevistado tem a possibilidade de discorrer sobre o tema proposto, sem condições pré-fixadas pelo pesquisador, sempre como uma conversa, ou seja, a pesquisa como uma relação entre sujeitos que buscam reconhecer o saber ${ }^{(10)}$.

A organização dos dados teve início com a transcrição e posterior ordenação das entrevistas. As participantes do estudo foram denominadas G1, G2 ... G8. Em seguida, realizou-se a classificação e a categorização das falas, com sínteses coincidentes e divergentes de ideias, e sua ligação, ou não, a alguma categoria.

Os dados foram examinados de acordo com a análise de conteúdo operacionalizada em três fases: pré-análise, exploração do material e tratamento dos resultados, conforme descrito em outros textos científicos $^{(10-11)}$. A análise dos dados ocorreu com a codificação das falas das entrevistadas. Os códigos foram agrupados pelas semelhanças de significados em categorias específicas.

Os aspectos éticos da pesquisa foram considerados, obedecendo-se a Resolução no 196/96 da Comissão Nacional de Ética em Pesquisa do Ministério da Saúde. O projeto de pesquisa foi aprovado pelo Comitê de Ética e Pesquisa da Universidade Estadual de Montes Claros, sob o Parecer Consubstanciado n. 973/2008, aprovado em 27.03.08.

\section{RESULTADOS}

Os falas a seguir apontam para aspectos relacionados à pergunta norteadora do estudo. Nos depoimentos, é possível observar o destaque às expectativas e necessidades das gestantes com relação ao pré-natal realizado pelo enfermeiro no âmbito da ESF:

Superou na atenção, que nem sempre os médicos dão. Talvez porque eles não têm tempo. (G4) 
Eu acho excelente! Não tenho nada a questionar, porque eu tive a confiança durante o atendimento desse profissional [enfermeiro]. (G8)

O bom atendimento, baseado na escuta do usuário e o bom desempenho são fatos apontados nos depoimentos a seguir:

Em muitas coisas que eu precisava, eles [enfermeiros] esclareceram. O médico já é muito rápido e o enfermeiro tem mais paciência. (G6)

[...] todas as dúvidas estão sendo esclarecidas e a consulta não ficou aquém do que eu esperava. (G7)

Em alguns depoimentos, ressalta-se o desempenho profissional no que concerne ao exame físico, às perguntas feitas e às orientações dadas, bem como à resolutividade das condutas:

Para mim, o pré-natal está perfeito. Não tenho nenhuma dúvida. Todas as dúvidas estão sendo esclarecidas e não ficou nada a desejar. (G1)

\section{[...] eles também examinam a nossa barriga. Eu achei} isso muito interessante. (G2)

Meu pré-natal anterior foi feito pelo médico. Ele não me examinava e não fazia muitas perguntas. Com o enfermeiro foi diferente. Ele me fez várias perguntas e pediu os exames que eu acho que devem ser pedidos na consulta de pré-natal. (G4)

Antes eu não gostava. É que era meu médico que estava sempre me atendendo, mas agora eu estou gostando. (G5)

Destaca-se ainda, nos depoimentos, o cuidado baseado na escuta, diálogo, atenção, respeito e confiança:

Eu gostei, achei que eles explicam bastante o que a gente tem dúvida. A consulta é bem demorada, diferente. (G1)

No início, eu fiquei em dúvida. Eu não acreditei, mas depois do atendimento eu tive mais confiança. Agora estou gostando muito. (G7)

As gestantes entrevistadas evidenciaram, em seus discursos, a dimensão profissional, por meio da qual se analisou a competência e a atenção do enfermeiro em relação às demandas, à eficácia e à quantidade dos serviços oferecidos:

Eu indicaria, pois estou achando ótimo. Eu diria a outras gestantes que o pré-natal realizado pelo enfermeiro é tão bom como o do médico. (G2)

Eu indicaria porque tenho uma amiga que me disse que o pré-natal feito pelo enfermeiro é melhor, pois ele é mais atencioso conosco. (G6)

\section{DISCUSSÃO}

Procedeu-se à análise dos discursos dos sujeitos daa pesquisa, divididos em categorias de acordo com os assuntos relatados. Em seguida, e conforme cada categoria, realizou-se um paralelo entre os discursos dos sujeitos, a literatura e a compreensão olhar do pesquisador.

\section{Consulta de enfermagem pré-natal: expectativas e necessidades}

O papel do enfermeiro em todos os níveis da assistência e, principalmente, na ESF, é de grande relevância. No que se refere à assistência pré-natal, ele deve mostrar à população a importância do acompanhamento da gestação na promoção da saúde, prevenção e tratamento de distúrbios, durante e após a gravidez, bem como informá-la dos serviços disponíveis ${ }^{(12)}$. Nesse contexto, ressalta-se a questão de se atender às expectativas da clientela, com particular atenção aos padrões de serviços e solução de queixas, problemas e outras necessidades, quando se refere à satisfação da cliente ${ }^{(13)}$.

Em algumas entrevistas, é evidenciado o bom atendimento, tendo por base a escuta ativa e o bom desempenho profissional, que propiciam o vínculo do binômio usuária-serviço de saúde. Esse vínculo aperfeiçoa o processo da assistência e proporciona aos profissionais a oportunidade de conhecer seus clientes. Permite, ainda, que os usuários do serviço de saúde aumentem sua autonomia e satisfação. Dessa forma, fica evidenciada uma preocupação com a humanização do atendimento, bem como com a forma de organização do serviço de saúde que leva em conta as necessidades do usuário ${ }^{(14)}$.

$\mathrm{O}$ profissional enfermeiro apresenta-se como 
elemento ativo da equipe de saúde, por exercer um papel educativo e contribuir para a ocorrência de mudanças concretas e saudáveis nas atitudes das gestantes, dos familiares e da comunidade, sempre em busca de bem-estar e qualidade de $\operatorname{vida}^{(1)}$. Ele deve, também, possuir sensibilidade humana, saber ouvir e permitir a participação do paciente no processo de identificação dos próprios problemas de saúde, estabelecimento de prioridades e planejamento das ações educativas e de saúde, que conduzem à promoção e à manutenção da saúde ${ }^{(15)}$.

\section{Satisfação das gestantes sobre a Consulta de Enfermagem pré-natal}

$\mathrm{Na}$ visão da clientela, percebe-se que os enfermeiros são identificados, entre os demais profissionais, como aqueles que proporcionam escuta ativa, mesmo diante da realidade de sobrecarga nas atividades diárias $^{(16)}$.

O exercício do saber-ser, saber-fazer, saberconviver representa comprometimento e resulta em boa atuação profissional. Com esse entendimento, os enfermeiros que desenvolvem atividades na assistência pré-natal têm compromisso com a saúde da clientela, e reconhecem, dessa forma, a saúde como direito individual e coletivo às condições dignas de vida e trabalho ${ }^{(17)}$.

A satisfação pode ser observada pela reação da usuária diante do contexto, do processo e do resultado global da experiência vivenciada. Além disso, vale registrar que as opiniões dos usuários também são influenciadas por diferentes situações, expectativas, antecedentes, ideias, valores e crenças acerca do atual quadro do sistema de saúde ${ }^{(1)}$.

As gestantes, ao serem questionadas sobre o atendimento, destacaram o ato de examinar e o esclarecimento de dúvidas como demonstrações de bom desempenho profissional e de responsabilização de quem presta assistência. O desempenho profissional é avaliado pela gestante, por meio do interesse demonstrado no exame físico, nas perguntas feitas e orientações dadas, bem como na resolutividade das condutas.

As usuárias expressam alto grau de satisfação em relação à dimensão relacional, em todos os seus atributos: respeito, consideração, escuta, compreensão, acolhida e gentileza por parte do profissional que a atende ${ }^{(17)}$.

O tempo de duração da consulta interfere no juízo de satisfação do usuário com o serviço de saúde: quanto maior a duração da consulta, menor a insatisfação ${ }^{(17)}$.Vale ressaltar que estudo realizado sobre satisfação com a duração da consulta apontou que $31,8 \%$ dos entrevistados preferem a consulta com duração de mais de 20 minutos.

Apesar das muitas barreiras impostas, a Consulta de Enfermagem vem crescendo em importância e atuando cada vez forte em áreas diferenciadas (Oncologia, Geriatria, Obstetrícia). Em outras palavras, é na consulta que o usuário busca a solução de seus problemas para recuperar o bem-estar ${ }^{(15)}$.

A busca pela resolutividade dos problemas, bem como a qualidade do atendimento recebido foram os pontos mais destacados quando avaliadas as expectativas do usuário com relação ao encontro com o profissional de saúde ${ }^{(1,18)}$. A responsabilidade do profissional para com o estado de saúde do usuário é um dos elementos essenciais para um efetivo acolhimento. Além disso, desperta no usuário sentimento de confiança em relação ao profissional que presta a assistência.

\section{Atenção à gestante de baixo risco: atuação que possibilita reconhecimento}

A enfermagem é caracterizada como uma arte e uma ciência de cuidar do indivíduo num aspecto biopsicossocial. O saber é considerado importante para que se estabeleça a autonomia profissional, à medida que se ressalta a mútua determinação entre saber e poder e que não haja uma relação de poder sem a constituição de um campo de saber, ao mesmo tempo em que um novo saber estabelece novas relações de poder ${ }^{(19)}$.

$\mathrm{Na}$ ótica da competência social, o saber-ser deve articular o saber-fazer, o saber-conviver e o saberaprender. Assim, compreende-se que, no desenvolvimento da assistência pré-natal, o enfermeiro procura estabelecer com a clientela o exercício dos saberes, ainda que de forma velada.

A construção do papel próprio possui relação com a construção da identidade profissional, o que significa a explicitação do que é específico do enfermeiro, ou seja, o saber e o fazer que caracterizam a Enfermagem $^{(19)}$. Nesse sentido, a Consulta de Enfermagem, voltada para o atendimento a gestantes de baixo risco, visa a oferecer assistência integral clínico-ginecológica e educativa, atentando para o aprimoramento do controle pré-natal, do parto e 
puerpério ${ }^{(20)}$. $\mathrm{O}$ enfermeiro se mostra, assim, como elemento ativo da equipe de saúde, ao executar a assistência à gestante no pré-natal.

\section{CONSIDERAÇÕES FINAIS}

Em um primeiro momento, a presença do enfermeiro desperta desconfiança e insegurança nas mulheres. No entanto, esses conceitos mudam na medida em que essas mulheres vivenciam uma experiência com o profissional enfermeiro e desenvolvem relação de confiança e segurança na capacidade desse profissional.

Assinala-se, ainda, que o elemento qualidade tende a modificar-se quando se trata de um serviço que leva em conta as reações da clientela e os resultados das várias experiências do cotidiano, tendo em vista demonstrar e aperfeiçoar sempre a assistência prestada pelo profissional. Nesta realidade, o desempenho do enfermeiro na sua interação com as clientes, no decorrer do acompanhamento do pré-natal e, em particular, da Consulta de Enfermagem, reflete o perfil da instituição e a qualidade da assistência prestada pelos profissionais de saúde.

Espera-se que este estudo propicie também reflexões para os gestores e profissionais da ESF, não somente na unidade em estudo como também em outras unidades, a fim de se buscar novas estratégias para a melhoria da qualidade dos serviços de pré-natal. E que a Enfermagem, enquanto ciência, busque com presistência o tão almejado reconhecimento de sua profissão.

\section{REFERÊNCIAS}

1. Cotta RMM, Marques ES, Maia TM, Azeredo CM, Schott M, Franceschini SCC, et al. A satisfação dos usuários do programa de saúde da família: avaliando o cuidado em saúde. Sci Med. 2005;15(4):227-34.

2. Pinheiro R. Integralidade e práticas de saúde: transformação e inovação na incorporação e desenvolvimento de novas tecnologias assistenciais de atenção aos usuários no SUS. Boletim ABEM. [Internet] 2003 [acesso em 20 abr2008]. Disponível: http://tinyurl. com/4ultaaw

3. Aguiar ACS, Moura ERF. Percepção do usuário sobre a atuação da equipe de daúde da família de um distrito de Caucaia-Ce. Rev Bras Promoc Saúde. [Internet] 2004; 17(04) [acesso em 30 mai 2008]. Disponível: http://tinyurl.com/4hw778t

4. Rosa WAG, Labate RC. Programa saúde da família: a construção de um novo modelo de assistência. Rev Latino-Am Enfermagem. [Internet] 2005;13(6) [acesso em 12 jun 2008]. Disponível: http://tinyurl. com/4fxhv5x

5. Ministério da Saúde (BR). O Programa Saúde da Família e a Atenção Básica no Brasil. Brasília: Ministério da Saúde; 2002.

6. Ministério da Saúde (BR). Pré-natal e Puerpério: atenção qualificada e humanizada - Manual Técnico. Brasília: Ministério da Saúde; 2005.

7. Minas Gerais (BR). Secretaria de Estado de Saúde de Minas Gerais. Atenção ao pré-natal, parto e puerpério. $2^{a}$ ed. Belo Horizonte: SAS/SES; 2006.

8. Brasil. Lei n. 7.498, de 25 de junho de 1986. Dispõe sobre a Regulamentação do Exercício da Enfermagem e dá outras providências. Diário Oficial da República Federativa do Brasil, Brasília, 26 jun. 1986. Seção 1:1

9. Lima YM, Moura MA. Consulta de enfermagem prénatal: a qualidade centrada na satisfação da cliente. R. de Pesq.: cuidado é fundamental. [Internet] 2005;9(1/2) [acesso em 01 nov 2008]. Disponível: http://tinyurl. com/4ogwkx 8

10. Minayo MCS. O desafio do conhecimento: pesquisa qualitativa em saúde. 8a ed. São Paulo: Hucitec; 2004.

11. Bardin L. Análise de conteúdo. $4^{\mathrm{a}}$ ed. Lisboa: Edições 70; 2006

12. Benigna MJC, Martins JL, Nascimento WG. Pré-natal no Programa Saúde da Família (PSF): com a palavra, os enfermeiros. Cogitare Enferm. [Internet]. 2004;9(2) [acesso em 30 out 2006]. Disponível: http://tinyurl. com/4ede412

13. Mirshawka V. Hospital: fui bem tratado, a vez do Brasil. São Paulo: Makron Books; 1994.

14. Lima MADS, Ramos DD, Rosa RB, Nauderer TM, Davis R. Acesso e acolhimento em unidades de saúde na visão dos usuários. Acta Paul Enferm. [Internet] 2007;20(1) [acesso em 22 jun 2008]. Disponível:http:// tinyurl.com/4ufysj7

15. Twardowski JJCS, Correa RC, Fávaro JR. A importância da consulta de enfermagem para idosos com problemas cardiovasculares. Rev Eletr Enf Uniandrade. 
[Internet] 2006 [acesso em 30 mai 2008]. Disponível: http://tinyurl.com/4n7pbgz

16. Oliveira CB, Ramos MC. O grau de satisfação da usuária gestante na assistência pré-natal nas unidades de saúde da família de Vitória. Cad Saúde Pública. 2007;15(2):241-56.

17. Lima YMS, Moura MAV. A percepção das enfermeiras sobre a competência social no desenvolvimento da assistência pré-natal. Esc Anna Nery. 2008;12(4):672-8.

18. Traverso-Yepez M, Morais NA. Reivindicando a subjetividade dos usuários da rede básica de saúde: para uma humanização do atendimento. Cad Saúde Pública. 2004;20(1):80-8.

19. Gomes AMT, Oliveira, DC. Estudo da estrutura da representação da autonomia profissional em enfermagem. Rev Esc Enferm USP. 2005;39(2):145-53.

20. Cunha MA, Mamede MV, DMG, Mamede FV. Assistência pré-natal: competências essenciais desempenhadas por enfermeiros. Esc Anna Nery. [Internet]. 2009;13(1) [acesso em 07 nov 2010]. Disponível: http://tinyurl.com/4kbkyzu doi: 10.1590/ S1414-81452009000100020 